ARTÍCULOS ORIGINALES

Rev Chil Salud Pública 2018 ,

Vol 22(2): 145-154
CECILIA MOLINA

Problemática de la Salud (Facultad de Ciencias Políticas y Sociales) y Especialización en Salud Pública (Facultad de Ciencias Médicas). Universidad Nacional de Cuyo. Mendoza, Argentina cmolina@isg.org.ar

Articulo recibido el 14/11/2017 Aceptado el 30/05/2018

\section{AUTOGESTIÓN HOSPITALARIA Y CAMBIOS EN EL MODELO ASISTENCIAL. LOS MÉDICOS EN LA IMPLEMENTACIÓN DE LA EXPERIENCIA EN MENDOZA, ARGENTINA ${ }^{a}$}

\author{
Hospital SElF-MANAGEMENT AND CHANGES IN THE CARE \\ model. Physicians in the implementation of the POlicy \\ in Mendoza, Argentina
}

\section{RESUMEN}

Propósito: Comprender y analizar el papel de los médicos en la implementación del modelo asistencial delineado en la política de descentralización hospitalaria en Mendoza, Argentina, entre 1990 y 2015.

Metodología: Estudio cualitativo, orientado por constructivismo, realizándose 30 entrevistas semiestructuradas a una muestra intencional de médicos en posiciones estratégicas en el Ministerio de Salud y en seis hospitales descentralizados de alta y mediana complejidad. Los datos se analizaron en base al Framework Analysis.

Resultados: Los relatos reflejan que la fijación de prioridades asistenciales de los hospitales está más influenciada por la capacidad de negociación de jefes médicos y directivos que por criterios sanitarios delineados desde el gobierno central. El modelo de cuidados progresivos afecta dispositivos tradicionales de socialización médica, como las revistas de sala. El trabajo en redes asistenciales es visualizado como responsabilidad del hospital por entrevistados de los centros menos complejos. En contraste, desde los grandes centros se concibe que la coordinación con atención primaria es competencia ministerial. La estandarización clínica es legitimada cuando los procedimientos se construyen de manera participativa. Si bien convive con sistemas promovidos por la administración, la vigilancia de las buenas prácticas se dirime en espacios de la profesión.

Conclusiones: Las creencias compartidas, fruto de la socialización profesional, operan como filtros a partir de los cuales los médicos en posiciones relevantes adoptan y/o adaptan valores e instrumentos del modelo asistencial que subyace a la descentralización hospitalaria. Las herramientas gestionarias que le son inherentes conviven pero no eliminan formas de autogobierno arraigadas en los servicios.

Palabras clave: Política de reforma sanitaria, hospitales de autogestión, modelo asistencial, implementación, profesión médica.

a. Este trabajo recupera parte de los resultados de la tesis de doctorado en Ciencias Sociales titulada: Entre lo técnico y lo político. Los médicos en la descentralización de los hospitales públicos de Mendoza, Argentina aprobada en diciembre de 2015. Agradezco a mis directores Federico Tobar y Ernesto Vivares sus generosas orientaciones. 


\section{ABSTRACT}

Purpose: To understand and analyze the role of physicians in the implementation of the care model outlined in the hospital decentralization policy in Mendoza, Argentina, between 1990 and 2015.

Methodology: A qualitative study, with a constructivist approach, was conducted. A total of 30 semi-structured interviews were carried out with an intentional sample of physicians in strategic positions in the Ministry of Health and from six decentralized high-and medium-complexity hospitals. Data were analyzed using Framework Analysis.

Results: Participants' account indicate that the setting of healthcare priorities for hospitals is more influenced by the negotiation capacity of medical and administrative directors than by sanitary criteria delineated by the central government. The coordination of healthcare networks is seen as a responsibility of the hospital by interviewees from less complex centers. In contrast, those interviewed from high complexity hospitals view the coordination effort with primary care as a ministerial responsibility. Clinical standardization is legitimized when it is constructed in a participatory manner. Best practices monitoring, while promoted by administrative systems, is ultimately resolved in clinical medical spaces.

Conclusions: Shared beliefs, which are the result of professional socialization, operate as criteria by which physicians in relevant positions adopt and / or adapt values and instruments of the care model that underlies hospital decentralization. The management tools that are inherent to it coexist but do not eliminate forms of self-government rooted in services.

Keywords: Health reform policy, Hospital self-management, Care model, Implementation, Medical profession

\section{INTRODUCCIÓN}

Las reformas sanitarias orientadas al mercado sostienen que el Estado tiene que regular antes que prestar servicios, promover la competencia entre financiadores y prestadores y focalizar sus esfuerzos en la población pobre. ${ }^{1,3}$ La concepción restrictiva del Estado que inspira la agenda neoliberal promueve como valor central la eficiencia. Esto explica la mira en los hospitales, que son los centros asistenciales que consumen la mayor parte de los presupuestos públicos en salud.

En Latinoamérica, en las últimas décadas, bajo el impulso de los organismos de asistencia técnica y financiera que gravitan en el sector, ${ }^{1,3}$ la mayoría de los gobiernos ensayaron fórmulas reformistas. Hospitales descentralizados (HD), empresas sociales del Estado, establecimientos de autogestión en red, organizaciones sociales de salud, son los nombres como se las conoce en el continente.

Estos proyectos pretenden remplazar la lógica burocrática que distancia a los formuladores de políticas de los implementadores; transfieren facultades a los hospitales para que, con cierta autonomía, puedan tomar decisiones y, al interior de los centros, buscan involucrar en las decisiones y los controles a los profesionales. Suponen, en suma, una doble transferencia de poder: desde el gobierno central a los hospitales y desde los hospitales a los servicios médicos.

El gobierno de Mendoza, en Argentina, impulsó la descentralización hospitalaria (DH) a principios de la década de los 90 del siglo pasado como parte de una batería de cambios en diferentes áreas del Estado provincial antes de que el hospital público de autogestión (HPA) fuera promovido a nivel nacional. ${ }^{4}$ Con algunas modificaciones, el proyecto mantiene vigencia.

Estudios previos llamaron la atención sobre las especificidades de la $\mathrm{DH}$ en Mendoza respecto de otras jurisdicciones del país, donde, en general, no implicó cambios significativos en la gestión de los hospitales. Destacan que el proyecto local se articuló a una reforma sectorial no limitada al ajuste fiscal, ${ }^{5}$ que incorporó diseños organizativos innovadores ${ }^{6,7}$ y que se 
vio favorecida por inversiones públicas que en la década de los 80 pusieron a los hospitales públicos en una relación de ventaja frente a privados. ${ }^{8}$ El papel de la comunidad médica en la organización de la actividad asistencial, que es una de las atribuciones que las normas provinciales les confieren a los $\mathrm{HD},{ }^{9,11}$ no ha sido analizado.

Cuestiones como las formas en que se definen las prioridades de los centros, los criterios que guían la actividad de los servicios, así como definiciones, en base al saber médico sanitario, sobre los lugares y maneras de distribuir la oferta y los estándares de calidad esperables conforman la dimensión asistencial de las políticas sanitarias. ${ }^{12,13}$ Este artículo analiza la implementación de la dimensión asistencial de la DH en Mendoza entre 1992 y 2015 desde la perspectiva de médicos participantes en distintos momentos del proceso. Tiene por objetivo identificar creencias, criterios de validación profesional y formas de conocimiento compartidas a partir de los cuales los médicos, en diferentes niveles del gobierno de salud, enmarcan la propuesta y posibilitan o no que ejes clave del modelo se conviertan en prácticas rutinarias y en procedimientos habituales en los hospitales.

\section{METODOLOGÍA}

Estudio cualitativo, orientado por el constructivismo. Como tal, entiende que las políticas se reformulan a medida que se ejecutan $^{14,15}$ y se aproxima a los médicos como profesionales-expertos que, en razón de su autoridad reconocida en el ámbito de la salud, modelan las políticas a partir de visiones compartidas que combinan sistemas de creencias, comprensiones causales y objetivos afines. Desde esas "epistemes" realidad, proporcionan y difunden conocimiento consensual para enfrentar problemas, proponen alternativas y desechan las que no consideran viables. ${ }^{16}$ Se construyó una muestra intencional de 30 participantes que conjugó criterios de posición y de reputación. Mediante el primero, se procuró atender los distintos niveles de gobierno involucrados en la $\mathrm{DH}$ : ministros, subsecretarios y directores del Ministerio de Salud (ASMS) y directores de hospitales, jefes de servicios asistenciales y de control de gestión de seis HD: tres hospitales de referencia provincial (HRP) y tres de los cuatro hospitales regionales (HR). El criterio reputacional, recomendado para estudiar elites médicas, ${ }^{17}$ posibilitó localizar a actores relevantes a partir de la técnica "bola de nieve" o cadena de referencias. Mediante ese procedimiento, se identificó a actores clave pidiendo a los entrevistados que mencionaran a otros que ellos consideraban influyentes en diferentes momentos de la política en estudio en función de su trayectoria política y/o científicoprofesional. Con la combinación de criterios de inclusión se buscó acotar los sesgos ${ }^{18}$ que presenta cada uno de los criterios muestrales por separado ${ }^{c}$. En la tabla 1 se caracteriza a los sujetos de estudio.

A todos los potenciales participantes se les explicitaron los objetivos del trabajo y a quienes consintieron se les garantizó el anonimato. Hubo negativa por parte de tres jefes de servicios y una clara predisposición a participar entre médicos que habían ejercido papeles relevantes en relación con la política en diferentes momentos del proceso.

La información se obtuvo mediante entrevistas semiestructuradas, cuyos ejes de indagación se construyeron en base al análisis de los artículos y capítulos de las normas y documentos nacionales ${ }^{4,19,21}$ y provinciales ${ }^{9,10,11}$ relativos a la $\mathrm{DH}$ que hacen referencia explícita a aspectos del modelo asistencial. Por ejemplo, los modos esperables de relación del HD con

\footnotetext{
b. Peter Hass vincula las dimensiones intelectuales y político-normativas mediante las que los expertos influyen en las políticas recuperando el término "episteme" propuesto por Michel Foucault. Con él se refiere a "una forma dominante de ver la realidad social, a un conjunto de símbolos y referencias, expectativas e intenciones compartidas por colecciones concretas de individuos que comparten una visión del mundo (o epistemes) que delimita, para sus miembros, la construcción adecuada de la realidad social" (Hass, 1992: 27). ${ }^{16}$ c. El criterio posicional puede dejar de lado a actores influyentes cuyas identidades no son públicamente conocidas o no necesariamente coinciden con su posición formal, mientras que, atendiendo exclusivamente al criterio reputacional, se puede saturar la muestra con personas que comparten la misma perspectiva (Tansey, 2007: 19). ${ }^{18}$
} 
la red de servicios aparecen enunciados en la ley provincial 6015/93 y en el decreto nacional 578/93 del HPA y son precisados en el decreto nacional 939/2000 y en el apartado referido al fortalecimiento del hospital público del Plan Federal de salud de 2004, al que adhirió la provincia.

Las preguntas se plantearon como invitaciones a hablar de aspectos de la política y se crearon oportunidades para que los entrevistados pudiesen debatir las ideas expresadas por sus pares y los supuestos del investigador. ${ }^{22}$ Todas las entrevistas fueron grabadas, con excepción de tres, por pedido de los entrevistados. En esos casos se tomaron notas. La duración promedio fue de 50 minutos, y los contextos de aplicación fueron los respectivos lugares de trabajo. Las entrevistas se transcribieron textualmente y se revisaron por investigadores ajenos al proceso. La información obtenida se procesó respetando los momentos previstos en el Analysis Framework. ${ }^{23}$ Se realizó primero una organización provisoria de resultados a partir de los ejes que guiaron la investigación y de los que, sin estar contemplados, emergieron en las entrevistas. Luego se realizó una codificación vertical para identificar ideas clave y cuestiones recurrentes. Se construyeron de manera simultánea y también provisoria categorías de análisis inductivas, a partir de datos que no se buscaron apriorísticamente y que, una vez tipificados, se incorporaron de manera sistemática a las siguientes entrevistas. Posteriormente, se separaron los datos de su contexto textual original y se etiquetaron secciones de datos agrupadas por temas y se produjo un índice ordenado para reportar la investigación ${ }^{23}$. Las categorías y subcategorías se presentan en la tabla 2.

\section{RESULTADOS}

\section{La definición de prioridades en el hospital}

La fijación de prioridades asistenciales aparece altamente influenciada por la capacidad de negociación de los jefes médicos y por las relaciones que forjan con los directivos. Si bien la descentralización pauta que los directorios (conducción colegiada del hospital) son el ámbito donde articular los lineamientos asistenciales del ministerio de salud con las prioridades de los HD, al momento de tomar decisiones, criterios informales prevalecen por sobre criterios sanitarios. Una creencia compartida por los médicos jefes es que el desarrollo de los servicios depende del "compromiso" personal con el trabajo; "de las afinidades", "de la fluidez en las relaciones de cada uno con la dirección". No tiene que ver con una visión del hospital y el sistema de salud en su conjunto.

Esta situación es reconocida y objetada por las autoridades ministeriales: "los hospitales toman sus decisiones con información de la que carecen los niveles centrales porque no se pensó en los controles que había que fijar antes de descentralizar" (AS-MS) y también por directores de los centros. Parte de estos últimos advierten consecuencias negativas para los usuarios. "Los jefes entusiastas piden, logran más cosas y los que no tienen entusiasmo piden menos y esos servicios no renuevan tecnología ni metodologías de trabajo. Y los pacientes no tienen la culpa de que les toque un jefe poco empujador" ( $\mathrm{AH}-$ HRP).

\section{De las parcelas médicas a la atención centrada en las personas}

La propuesta de remplazar la atención fragmentada según especialidades por una forma organizativa cuyo eje sea la intensidad de cuidados que demanda el paciente desafía el ordenamiento histórico del hospital distribuido en base a clasificaciones médicas, las rutinas de formación y la concepción de las camas como propiedad de los servicios.

El peso del modelo divisionario por especialidades es mayor en los hospitales complejos, donde el componente educativo es importante. En estos centros, los cuidados progresivos impulsados por la $\mathrm{DH}$ conspiran contra dispositivos de socialización médica, como la revista de sala, e interfieren en el objetivo de profundizar en la propia especialidad. "Cuando se saturó un servicio y por ejemplo queda una cama libre en neurocirugía, entra un paciente clínico y viene el clínico a seguirlo. Esto se hace. El problema es que como médico, más allá de ser médico, estás haciendo una promoción muy especifica en tu especialidad, y tener a un 
paciente en otro lugar te hace dejar de hacer la tarea en la que te estás formando". (JSA-HRP).

La forma habitualizada de organizar el trabajo asistencial a partir de las especialidades, reflejada en la estructura organizacional, restringe la aplicación del modelo: "En esto no se ha avanzado todo lo que uno quisiera y no por culpa de la gestión sino por culpa nuestra, de los médicos (...) Antes los servicios estaban encerrados. Era: clínica médica tiene tantas camas, se acabaron las camas, entonces me voy. Desde hace unos años cambió la óptica: las camas son del hospital, no de los servicios". (JSA- HR).

\section{Autonomía hospitalaria versus trabajo en redes}

En general, los médicos de los HRP consideran que la coordinación con atención primaria compete al gobierno central. En consecuencia, las iniciativas de conexión son puntuales y el hospital absorbe problemas que, en teoría, deberían tener resolución en otros niveles, resintiendo la operatoria del propio centro. "Lo ideal sería que cuando yo le dé de alta al paciente, se fuera con una referencia al centro de salud. Ha habido intentos... pero bueno, en muchos casos el paciente viene y dice: no tengo turno, no me atendieron bien y lo terminás comprando". (AH- HRP).

En contraste, los médicos que se desempeñan en HR interpretan que la coordinación entre quienes actúan en un mismo territorio a través de consejos locales de salud es un mecanismo válido para avanzar en la continuidad asistencial. A partir de esta creencia, acuerdan carriles de acceso y criterios comunes de derivación y de circulación de la información clínica. No depositan esa tarea en el gobierno central.

Una idea compartida es que la falta de mecanismos organizacionales de coordinación entre niveles asistenciales se compensa mediante las relaciones informales "entre colegas". La fuerza de estas interacciones está sujeta a atribuciones de autoridad mutuas y resulta de intercambios previos en espacios de socialización profesional, que generan confianza y posibilitan la conexión. "Si el médico del primer nivel se formó en el hospital, si se vinculó con los que son los maestros de las particularidades, se establece una relación de respeto mutuo... Esto es esencial para favorecer las referencias y contrarreferencias". (AS-MS).

\section{Entre el arte y la estandarización: las guías de práctica clínica}

Los protocolos son aceptados en la medida que se construyen o validan participativamente en espacios clave de socialización médica: los comités de docencia, las reuniones científicas o el sistema de formación. Por el contrario, tienden a ser impugnados cuando los impulsa la administración. "Las normas que calan son las que son fruto del debate en el hospital". (JSMHRP).

En las áreas clínicas, el uso de guías de práctica se considera un requisito que va de la mano de la formación profesional. "Ser un hospital escuela nos obliga a tener guías de diagnóstico y tratamiento... nos obliga a tener una forma sistematizada de actuar" (JSM HRP).

El trabajo en base a guías y el registro de las prácticas son percibidos como factores que dan consistencia al hospital público pero también como un mecanismo de contención que preserva a los médicos de las amenazas a las que se sienten expuestos en el actual contexto del ejercicio profesional. "Cuando yo me formé, hablar de juicio por mala praxis era rarísimo y no estaba en nuestra mente pedir tal o cual cosa por miedo a lo que digan los abogados. Las cosas han cambiado y, en definitiva, seguir un protocolo y trabajar en equipo hoy pesa". (JSM- HR).

No obstante la aceptación de cierta necesidad de estandarizar las prácticas, los médicos en distintas posiciones creen que su aplicación inflexible pone en riesgo la eficacia del juicio clínico. Se defiende la idea de la medicina como "ciencia y arte" "... las normas no están para fijar conductas: son el marco para justificar cuándo salirse de ellas". (AH- HRP).

Una idea compartida por médicos en diferentes posiciones $-y$ que recibe objeciones por quienes ejercen funciones de control de gestión- es que las decisiones clínicas se fundan en una especie de "sentido común" profesional, en una forma de aprender entre pares que unifica criterios, orienta las intervenciones y exime a quienes las practican de intromisiones externas. Es el cuerpo de conocimientos que transmite 
la medicina y no la organización hospitalaria quien provee las reglas de comportamiento que se consideran apropiadas. "Nos manejamos con normas, pero si me preguntás: ¿lo tenés en un papel? Y no, son cosas básicas... sería como pedirle a un sacerdote que lleve escrito el Padre Nuestro en el bolsillo". (JSA- HRP).

\section{La calidad asistencial: un asunto médico}

La definición de los atributos de la asistencia se dirime y está sujeta a criterios validados por los profesionales por afuera de las fronteras físicas del hospital. Si bien en unos pocos casos convive con sistemas promovidos por la administración, la evaluación de la calidad es considerada una actividad concerniente a los servicios. "Lo ideal sería que la gerencia releve los servicios y diga qué mínimos se necesitan para tener calidad, pero en realidad esto lo hace el jefe de servicio y cada uno sigue su criterio". (JSA- HRP).

En general, se comparte que la combinación de la actividad asistencial con la docencia garantiza la calidad de los cuidados. Como sucede con la adopción de protocolos, la vigilancia de las buenas prácticas se dirime en espacios de socialización profesional. "La formación te tira para arriba la calidad del sistema... La capacitación de los colegas exige muchísimo". (AS-MS).

La evaluación de la calidad asistencial que va más allá de los atributos técnico- científicos tiene escasa cabida en el discurso médico. Excepcionalmente los criterios para relacionarse con los enfermos, para transmitirles información clínica, son explícitamente pautados desde los servicios.

\section{DISCUSIÓN}

Los resultados expuestos sugieren que la mayor autonomía de los hospitales públicos no necesariamente añade racionalidad a las decisiones que improntan sobre la actividad asistencial de los centros ${ }^{24,2}$ ni las hace más receptivas a las necesidades sanitarias de las poblaciones a las que se referencian. ${ }^{25-27}$

Los médicos en diferentes posiciones del gobierno de la salud en Mendoza comparten que antes que a misiones políticamente definidas, gran parte de las demandas de crecimiento de los servicios asistenciales en los HD responden a las inquietudes de quienes trabajan en la organización. Como lo reportan otros análisis en el continente ${ }^{28,30}$, coinciden en la sustitución de la atención fragmentada en base a especialidades por un modelo de cuidados progresivos, que ponga en el centro de la organización las necesidades de los pacientes es fuente de tensiones entre servicios y entre los servicios y los directivos.

Estudios europeos que ponen foco en el papel de los médicos en las reformas hospitalarias orientadas al mercado coinciden en que la profesión mantiene considerable influencia en los contenidos de las políticas asistenciales y en los estándares de atención aun cuando las reformas procuren acotar márgenes de autonomía médica. ${ }^{31-33,17}$

En el proceso aquí analizado se observa que la estandarización de las prácticas clínicas y los controles de calidad técnica, siempre que sean validados profesionalmente, son altamente congruente con la episteme médica. No son percibidos como fuerzas externas y en la medida que los jefes de servicios ponen esas herramientas bajo su control, las utilizan activamente, las adoptan como recurso organizacional $y, a$ veces, como mecanismos para preservar la profesionalidad. ${ }^{31}$ La mayoría defiende poder elegir entre alternativas terapéuticas, atendiendo a las particularidades de los pacientes, al contexto en que sus prácticas tienen lugar ${ }^{34}$ y a los aprendizajes que resultan de sus espacios de socialización profesional. ${ }^{35}$

En contaste con los enunciados de la $\mathrm{DH},{ }^{10,20,21}$ el proyecto no es valorado como una oportunidad para coordinar acciones con el conjunto de la red de servicios y de ese modo garantizar la continuidad de la atención. La integración entre niveles asistenciales a través del trabajo en redes se interpreta mayormente como un problema que excede al hospital. ${ }^{36}$

Los modos en que se implementan los cambios asistenciales han sido poco considerados en los análisis sanitarios ${ }^{13}$ en general y los proyectos de autonomía hospitalaria en Latinoamérica. Se han estudiado más las insuficiencias del modelo para superar la fragmentación de los sistemas de salud y las inequidades en el acceso ${ }^{1,6,7,27,28}$ que 
las repercusiones de las nuevas modalidades gestionarias en la provisión de servicios y el papel de los equipos de salud en los procesos. ${ }^{26,36,37}$ Los hallazgos que aquí se presentan pretenden ser una contribución en este sentido.

La perspectiva constructivista que orientó este estudio se aleja de los enfoques racionales o prescriptivos que establecen una demarcación clara entre formulación e implementación de las políticas de salud e intenta esclarecer de qué modo las ideas compartidas a partir de las cuales los expertos enmarcan las políticas, las redefinen en la ejecución. Si bien el diseño no pretende ni permite generalizar resultados, posibilita explorar actores y procesos que median en la consecución los enunciados. ${ }^{38,23}$

La muestra presentada procuró incluir a una amplia gama de informantes con una amplia gama de experiencias ${ }^{39}$ en el período analizado, atendiendo a hallazgos previos que sugieren diferencias en la adopción de propuestas de reforma hospitalaria entre médicos en posiciones gerenciales y clínicas, ${ }^{40,32}$ pero tiene limitaciones. Por un lado, sobre 14 HD provinciales, se circunscribió a los 6 de mayor capacidad resolutiva, mayor gravitación en sus áreas de influencia y donde el componente educativo es más relevante. Por otro lado, al centrarse en médicos en posiciones de autoridad, no ahondó con lo que sucede puertas adentro de los servicios, cuando la $\mathrm{DH}$ también pretende afectar procesos de reasignación de autoridad a ese nivel. ${ }^{7,12}$ De allí que sería deseable encarar nuevos trabajos que exploren los mismos ejes entre médicos asistenciales. Asimismo, atendiendo a la permeabilidad del proyecto para contratar a profesionales en base a criterios de mercado, se debería profundizar cómo impacta la progresiva estratificación de las especialidades médicas en la dinámica de trabajo asistencial de los DH. En cuanto a los diseños, sería importante desarrollar estudios comparativos que permitan dar cuenta del devenir de las relaciones entre reformas hospitalarias y profesión médica ${ }^{39,31}$ en sistemas de salud altamente segmentados en el financiamiento y provisión como los latinoamericanos, donde el pluriempleo y la no profesionalización de la función directiva están extendidos.

\section{CONCLUSIONES}

Este estudio intenta echar luz sobre los modos en que los actores locales, conjugando experticia profesional y cierta capacidad de decisión política, adoptan y/o transforman las ideas y estrategias de reforma global en las instancias subnacionales en las que se diseñan y ejecutan las políticas

La adopción por parte de los médicos de los nuevos conceptos e instrumentos contemplados en la dimensión asistencial de DH está mediada por creencias causales, por formas comunes de conocer validadas más allá de los límites del hospital y por jerarquías intraprofesionales que no necesariamente se condicen con las posiciones que se ocupan en la estructura formal del gobierno de la salud. Desde esas coordenadas se dirime qué cuestiones contempladas en los diseños de las políticas es posible incorporar, cuáles son eludidas y cuáles demandan ser negociadas en el transcurso de la implementación.

Tabla N $N^{\circ} 1$ Perfil de los entrevistados

\begin{tabular}{|l|c|}
\hline $\begin{array}{l}\text { Principal función identificada para la } \\
\text { entrevista }\end{array}$ & N \\
\hline Autoridad sanitaria provincial (AS) & 7 \\
\hline Autoridad hospital (AH) & 9 \\
\hline Jefe de servicio asistencial (JSA) & 9 \\
\hline Jefe de unidad de control de gestión (JCG) & 3 \\
\hline $\begin{array}{l}\text { Dirigente organización de intereses } \\
\text { médicos (DOM) }\end{array}$ & 2 \\
\hline Total & 30 \\
\hline Ámbito de desempeño & \\
\hline Ministerio de Salud (MS) & 7 \\
\hline Hospital de referencia provincial (HRP) & 16 \\
\hline Hospital regional (HR) & 8 \\
\hline Total & 30 \\
\hline Período de vinculación con el proyecto & \\
\hline Menos de 5 años & 1 \\
\hline Entre 6 y 10 años & 9 \\
\hline Entre 11 y 15 años & 13 \\
\hline Entre 16 y 20 años & 4 \\
\hline Más de 20 años & 5 \\
\hline Total & 30 \\
\hline
\end{tabular}


Tabla N ${ }^{\circ} 2$ Categorías y subcategorías de análisis

\begin{tabular}{|c|c|}
\hline \multirow{5}{*}{$\begin{array}{l}\text { Fijación de prioridades } \\
\text { asistenciales en los hospitales }\end{array}$} & Valores que involucra \\
\hline & Adecuación a lineamientos del gobierno central \\
\hline & Liderazgo de directivos \\
\hline & Liderazgo de los jefes de servicio \\
\hline & $\begin{array}{l}\text { Consecuencias de los procesos decisorios en la actividad } \\
\text { asistencia }\end{array}$ \\
\hline \multirow{2}{*}{$\begin{array}{l}\text { Implantación del modelo } \\
\text { de cuidados integrados }\end{array}$} & Aspectos que lo favorecen \\
\hline & Obstaculizadores \\
\hline \multirow{3}{*}{$\begin{array}{l}\text { Papel del hospital en } \\
\text { la red asistencial }\end{array}$} & Aspectos que favorecen la referencia y la contra-referencia \\
\hline & Obstáculos en la referencia y la contra- referencia \\
\hline & $\begin{array}{l}\text { Nivel de responsabilidad en la coordinación entre niveles } \\
\text { asistenciales }\end{array}$ \\
\hline \multirow{4}{*}{$\begin{array}{l}\text { Organización y control } \\
\text { del trabajo médico }\end{array}$} & Uso de guías de práctica clínica \\
\hline & Uso de sistemas de información \\
\hline & Políticas de calidad técnica y percibida \\
\hline & $\begin{array}{l}\text { Gravitación del hospital escuela en la actividad asistencial } \\
\text { y la calidad de la atención }\end{array}$ \\
\hline
\end{tabular}

\section{REFERENCIAS BIBLIOGRÁFICAS}

1. Ugalde A, Homedes N. América Latina: la acumulación de capital, la salud y el papel de las instituciones internacionales. Salud Colect. 2007;3(1):33-48.

2. Homedes N, Ugalde A. Las reformas de salud neoliberales en América Latina: una visión crítica a través de dos estudios de caso. Rev Panam Salud Pública. 2005;17 (3):210-220.

3. González N, Nin C. Entrevista con Asa Cristina Laurell. Tiempos de resistencia. Por el derecho a la salud. ConCienciaSocial [en línea]. 2018 [consultado el 20/05/2018];1(2):179-187. Disponible en: https://revistas.unc.edu.ar/index.php/ ConCienciaSocial/

4. Hospitales. Registro de hospitales de autogestión. Decreto 578. Publicado en el Boletín oficial del 1 de abril de 1993. Número 27614, República Argentina.

5. Repetto F, Potenza Dal Maseto F.
Problemáticas institucionales de la política social Argentina: más allá de la descentralización. Ponencia presentada en el VIII Congreso Internacional del CLAD sobre la Reforma del Estado y de la Administración pública, Panamá, 28-31 Oct. 2003.

6. Sojo A. Argentina: el gran abanico de la descentralización hospitalaria. En: Sojo A. comp. Reformas de gestión en salud en América Latina: los cuasi mercados de Colombia, Argentina, Chile y Costa Rica. Serie Políticas sociales No39. Santiago de Chile: CEPAL; 2000. p. 39-42.

7. Tobar F. Alternativas para la financiación hospitalaria en Argentina. Medicina y Sociedad. 1998;21(1):29-40.

8. Belmartino S, Bloch C, Báscolo E. Reforma de la atención médica en escenarios locales. 1990-2000. Buenos Aires: Lugar Editorial; 2003.

9. Régimen de descentralización del hospital público. Carreras de profesionales de la salud. Modificación de diversas normas. Ley 
6015. Publicada en el Boletín Oficial 17 de junio de 1993, Provincia de Mendoza, Argentina.

10. Régimen de descentralización del hospital público. Reglamentación de la Ley 6015. Decreto 2310. Publicada en el Boletín Oficial 1 de febrero de 1994, Provincia de Mendoza, Argentina.

11. Régimen de descentralización del hospital público. Modificación de la Ley 6015. Ley 7099. Publicada en el Boletín Oficial 17 de marzo de 2003, Provincia de Mendoza, Argentina.

12. Tobar F. ¿Qué aprendimos de las reformas en salud? Evidencias de la experiencia internacional y propuestas para Argentina. Buenos Aires: Fundación Sanatorio Güemes; 2010.

13. Chiara M. Salud, política y territorio en el Gran Buenos Aires. Los Polvorines: Universidad Nacional de General Sarmiento; 2012.

14. Parsons W. Políticas públicas: una introducción a la teoría y a la práctica del análisis de políticas públicas. México: FLACSO. Sede académica de México; 2007.

15. Bernan P. El estudio de la macro y microimplementación. En: Aguilar Villanueva L. ed. La implementación de las políticas. México: Miguel Ángel Porrúa Grupo editorial; 1993. p 281-322.

16. Haas P. Introduction: epistemic communities and international policy coordination Source: Int. Organ. 1992;46(1): 1-35.

17. Lewis J. Being around and knowing the players: Networks of influence in health policy. Soc Sci Med. 2006;62:2125-2136.

18. Tansey O. Process Tracing and Elite Interviewing: A Case for Non-probability Sampling. PS-Polit Sci Polit. 2007;40(4):123.

19. Argentina. Ministerio de Salud y Acción Social de la Nación. Hospital público de autogestión. Marco conceptual, estrategias e instrumentos operativos. República Argentina; 1996.

20. Créase el Régimen de hospitales públicos de gestión descentralizada. Objetivos. Decreto 939. Publicado en el Boletín Oficial el 24 de octubre de 2000, República Argentina.

21. Chile. Ministerio de Salud de la Nación.
Consejo Federal de Salud de la República Argentina. Bases del plan federal de salud 2004-2007 [en línea]; 2004. p.32-35 [consultado el 26 de sept 2014]. Disponible en: $\quad$ http://www.ossyr.org.ar/PDFs/2004_ Plan_Federal_Salud.pdf

22. Erasmus E, Gilson L. How to start thinking about investigating power in the organizational settings of policy implementation. Health Policy Plan. 2008;23(5): 361-368.

23. Srivastava A, Thomson SB. Framework Analysis: A Qualitative Methodology for Applied Research. Note Policy Research. JOAAG. 2009;4(2):73-79.

24. Tobar F. ¿Hacia dónde va el hospital? Desafíos y dilemas en la gestión de hospitales [en línea] [consultado el 22 de mayo 2018] Disponible en: https://es.scribd.com/ document/378932960/Tobar-2008-HaciaDonde-Va-el-Hospital.

25. Barasa E, Molyneux S, English M, Cleary S. Setting healthcare priorities in hospitals: a review of empirical studies. Health Policy Plan. 2014;1(11):386-396.

26. Méndez C, Miranda C, Torres M, Márquez M. Política de autogestión hospitalaria en Chile: percepciones de los tomadores de decisiones. Rev Panam Salud Pública. 2013;33(1):47-53.

27. Arroyo Laguna J. La autonomización de hospitales públicos y sus consecuencias sobre la demanda de atención de salud, Lima, 1988-1997. Rev Panam Salud Publica.1999;6(5):301-310.

28. Artaza Barrios O, Méndez CA, Holder Morrison R, Suárez Jiménez JM. Redes integradas de servicios de salud: el desafío de los hospitales. Santiago Chile: OPS/OMS; 2011.

29. Schiavone MA, Mazza P, Cordero L. Internación por cuidados progresivos: propuesta metodológica para su implementación en un hospital general de agudos. Rev Asoc Med Argentina 2011;124(3):17-23.

30. Jarillo Soto E. La profesión médica. Una construcción de la ideología profesional. En Jarillo Soto E. Guinsberg E, editores Temas y desafíos en salud colectiva. Buenos Aires: 
Lugar Editorial, 2007. p. 327-346.

31. Kuhlmann E, Burau V, Correia T, Lewandowski R, Lionis C, Noordegraaf M. et al. "A manager in the minds of doctors:" a comparison of new modes of control in European hospitals BMC Health Serv Res [en línea]. 2013246 [consultado el 30 de agosto 2015];13,246-. Disponible en http://www.ncbi.nlm.nih.gov/pmc/articles/ PMC3702431/.

32. Neogy I, Kirkpatrick I. Medicine in management: lessons across Europe. Leeds: Centre for Innovation in Health Management Policy Report, University of Leeds; 2009

33. Ugalde A, Homedes N. Las reformas neoliberales del sector de la salud: déficit gerencial y alienación del recurso humano en América Latina. Rev Panam Salud Publica. 2005; 17(3):202-209.

34. Lemp S, Calvo EE. ¿Aceptación o rechazo de la estandarización clínica? médicos chilenos hablan de las guías clínicas y canastas de prestaciones. Salud Colect. 2012;8(1):61-68.

35. Deom M, Agoritsas P, Bovier P, Perneger T. What doctors think about the impact of managed care tools on quality of care, costs, autonomy, and relations with patients $B M C$ Health Serv Res [en línea]. 2010 [consultado el 1 de enero 2014];10(331). Disponible en: http://www.biomedcentral.com/14726963/10/331

36. Méndez C, Miranda C, Torres M, Márquez M, Matthews T. Implementación de la política de hospitales autogestionados en Chile: percepción de los profesionales hospitalarios. Rev Chil Salud Pública. 2012;16(3):247-255.

37. Carrioni CA, Hernández ML, Molina G. La autonomía de las instituciones prestadoras de servicios de salud : más un ideal que una vivencia institucional. Rev Fac. Nac Salud Pública. 2007;25(2):75-84.

38. Pope C, Ziebland S, Mays N. Qualitative research in health care. Analysing qualitative data. BMI. 2000; 320:114-116.

39. Spehar I, Frich J, Kjekshus LE. Clinicians in management: a qualitative study of managers' use of influence strategies in hospitals. BMC Health Serv Res [en línea]. 2014 [consultado el 10/11/2015];14(251). Disponible en: http://www.biomedcentral. com/content/pdf/1472-6963-14-251.pdf. [

40. Witman Y, Smid G, Meurs P, Willems D. Doctor in the lead: balancing between two worlds. Organization. 2010;18(4):477-495. 